\title{
Non-destructive characterization of wire arc sprayed cylinder coatings using laser- excited lock-in thermography measurements
}

\author{
by J. Arbogast*,**, R. Schaller*, B. Schilder*, S. Lindemann*, M. Rohde ${ }^{\star \star}$, H.J. Seifert** \\ * Mercedes-Benz AG, Stuttgart, Germany, jannik.arbogast@daimler.com \\ **Karlsruhe Institute of Technology, Karlsruhe, Germany
}

\begin{abstract}
Laser-excited Lock-In Thermography is used to evaluate the thermal diffusivity of thermally sprayed cylinder bore coatings of passenger car engines. Further, microstructural analysis as well as adhesion testing of the investigated cylinder bore coatings are acquired to evaluate microstructural and mechanical properties. Investigation of the interaction between thermal diffusivity, microstructural and mechanical properties disclose major correlations between the observed quantities. The application of thermal diffusivity measurements as a non-destructive testing method of adhesion strength in thermally sprayed cylinder bore coatings is discussed in this work.
\end{abstract}

\section{Introduction}

In order to improve the efficiency of modern powertrains, the NANOSLIDE ${ }^{\mathrm{TM}}$ process chain was developed by Mercedes-Benz, using twin wire arc spraying and surface-finish through honing to reduce the frictional losses between pistons and cylinder running surface by up to $50 \%$ [1]. To guarantee life-long functionality of the combustion engine, mechanical properties such as adhesion strength of the coating to the substrate may be monitored during series production. By now, adhesion strength can only be carried out by destructive off-site testing, while it can be shown that the microstructure of thermally sprayed cylinder bore coatings directly influences the coatings' adhesion [2]. Since nondestructive thermal diffusivity measurements and microstructural analysis of such cylinder bore coatings have revealed direct influences of the microstructure on the thermal diffusivity values [3], this work investigates the interaction of thermal diffusivity, microstructure and adhesion strength of twin-wire arc spray cylinder coatings.

\section{Materials - NANOSLIDE ${ }^{\mathrm{TM}}$}

The NANOSLIDE ${ }^{\mathrm{TM}}$ process chain combines mechanical roughening the cylinder bore of the aluminum crankcase followed by twin wire arc spraying and mechanical machining including honing of the low-alloyed carbon steel coatings [1]. Fig. 2A. shows the morphology of a thermally sprayed cylinder bore coating including the typical lamellar structure of partially separated splats and porosity [4].

\section{Methods}

Measurements and samples of all the following methods are performed at positions $10 \mathrm{~mm}, 40 \mathrm{~mm}, 70 \mathrm{~mm}$, $100 \mathrm{~mm}$ and $130 \mathrm{~mm}$ under the cylinder head sealing surface for all cylinder liners at the same angle position. The described analysis were performed on three different crankcases of type OM656 (Mercedes-Benz AG, Stuttgart, Germany).

\subsection{Thermal wave interferometry (TWI)}

Thermal diffusivity $\alpha$ of thermally sprayed coatings are measured by thermal wave interferometry [5]. A periodically amplitude-modulated laser source $(250 \mathrm{~W}$ maximum power, $938 \mathrm{~nm})$ with frequency $\mathrm{f}$ is used to generate thermal waves within the coating material. Partial reflections of the thermal wave at the interface between layer and substrate lead to interference with incoming thermal waves causing a phase shifted temperature modulation on the layer surface compared to the modulated heat source [5]. The described phase shift $\varphi$ can be written as

$$
\varphi(f)=\tan ^{-1}\left(\frac{-2 R \cdot \exp (-2 \eta \sqrt{f}) \cdot \sin (2 \eta \sqrt{f})}{1-(R \cdot \exp (-2 \eta \sqrt{f}))^{2}}\right) \quad \text { with } \quad \eta=d \cdot \sqrt{\pi / \alpha}
$$

where $\mathrm{R}$ describes the reflection coefficient of the thermal wave and d describes the thickness of the coating layer. An optical system including an infrared camera system is used to detect the phase shifted surface temperature modulation, which is synchronized to the excitation laser source by a signal generator. TWI measurements are performed at frequencies ranging from $5 \mathrm{~Hz}$ to $150 \mathrm{~Hz}$ and a laser spot size of $6.7 \mathrm{~mm}$. Nonlinear least square fitting is used to fit Eq. (1) to the measured phase values and calculate the thermal diffusivity values. Thickness values $d$ of the coating layer are measured with X-ray computed tomography. 


\subsection{Microstructural analysis}

Light microscopy images of cross sections are used to achieve microstructural analysis of the observed thermally sprayed coatings. Image analysis is carried out using an algorithm based on Python package OpenCV to detect and quantify observed defects in the cylinder coating. Influences of the detected defects on the thermal diffusivity are evaluated by calculating the additional path length of thermal diffusion due to amount, size and shape of the detected defects [7].

\subsection{Precision adhesion testing (PAT)}

Precision adhesion testing (PAT) is used to measure the adhesion strength of the thermally sprayed coatings to the substrate. Therefore, test elements with $8 \mathrm{~mm}$ diameter are placed on the coating surface and pulled off hydraulically. All shown values are normalized on the minimum required adhesion strength of cylinder coatings in Mercedes-Benz engines. Further, breakage is analyzed quantitatively regarding remaining coating material on the aluminum substrate by scanning electron microscope (SEM).
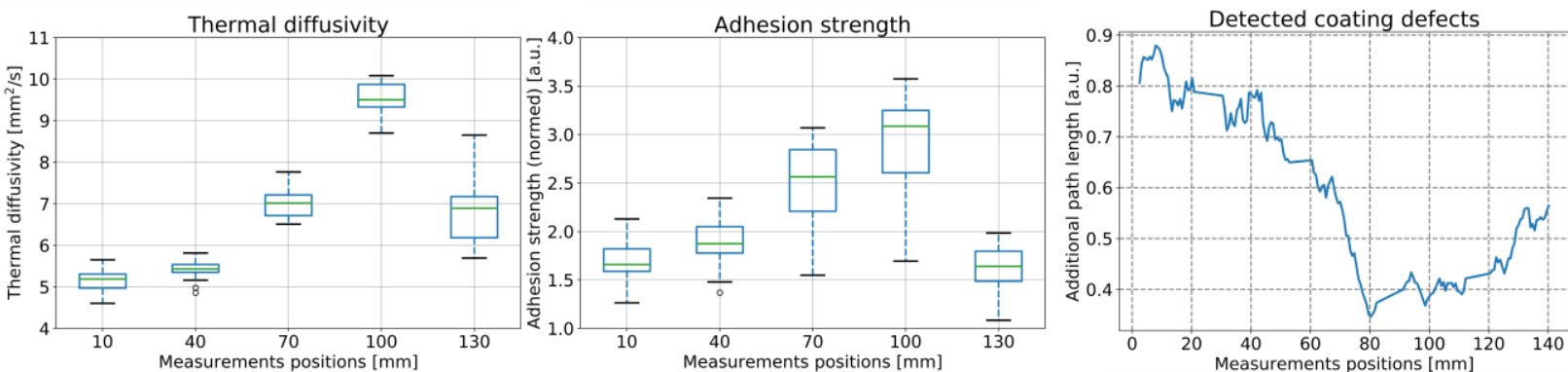

Fig. 1. Left: TWI thermal diffusivity measurements of the cylinder coating, Middle: Adhesion strength measurements of the cylinder coating normed on the minimum required adhesion strength. Right: Influences of segmented coating defects on thermal diffusion processes. Boxplots: The green lines indicate the median of the underlying data; the boxes indicate the upper/lower quartile, the whiskers are set to $1.5 \mathrm{x}$ interquartile range.
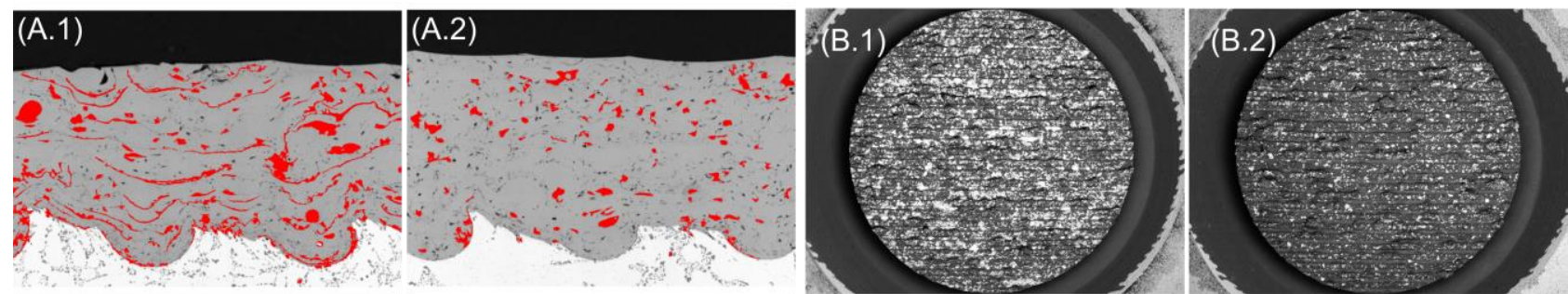

Fig. 2. A: Cross section images including the segmented coating defects highlighted in red (A.1: $40 \mathrm{~mm}, A .2: 100 \mathrm{~mm})$. $B$ : SEM images of the breakage $(B .1: 40 \mathrm{~mm}, B .2: 100 \mathrm{~mm})$. Remaining coating material is shown in light grey; aluminium substrate is shown in dark grey.

\section{Results}

TWI measurements show strong dependencies of the thermal diffusivity on the measurement position along the cylinder liner. Comparing measurement positions $10 \mathrm{~mm}$ and $100 \mathrm{~mm}$, the median of the thermal diffusivity almost doubles from $5.2 \mathrm{~mm}^{2} / \mathrm{s}$ to $9.5 \mathrm{~mm}^{2} / \mathrm{s}$ (Fig. 1. Left). Further, measured adhesion strength values vary along the cylinder liner (Fig. 1. Middle) and show a similar relative behavior as the thermal diffusivity values. Comparing the obtained median values at positions $10 \mathrm{~mm}$ and $100 \mathrm{~mm}$, adhesion strength rises from 1.66 to 3.09. Fig. 1. Right depicts the additional path length of thermal diffusion processes due to detected defects within the coating material based on the cross section images. While a strong decline of detected defects towards measurement position $80 \mathrm{~mm}$ can be found, subsequently a comparably moderate increase towards $140 \mathrm{~mm}$ is shown. Two cross section images of the cylinder coating at positions $40 \mathrm{~mm}$ and $100 \mathrm{~mm}$ including segmented defects can be found in Fig. 2A, while corresponding SEM images of the breakage after adhesion testing at the same positions are shown in Fig. 2B. Quantitative analysis of the SEM images reveals a decline of remaining coating material on the substrate after adhesion testing from $26.5 \%$ to $10.3 \%$ for positions $40 \mathrm{~mm}$ and $100 \mathrm{~mm}$, respectively.

\section{Discussion}

Thermal diffusivity measurements, adhesion testing as well as defect detection measurements show variations along the cylinder liner. While thermal diffusivity and adhesion values show lowest values in measurement positions $10 \mathrm{~mm}$ 
and $40 \mathrm{~mm}$, both quantities peak at measurement position $100 \mathrm{~mm}$ followed by a strong decline towards position $130 \mathrm{~mm}$. Analyzing the microstructure of the cylinder coating, enhanced amount of defects indicate a direct correlation of both thermal diffusivity and adhesion to the coatings' microstructure. While laterally expanded splat interfaces act as thermal barriers reducing the thermal diffusivity orthogonal to the coating surface [8], the described splat interfaces may also reduce the interlamellar bonding of the cylinder coating $[2,4]$. Therefore, an indirect correlation of adhesion strength to the thermal diffusivity of the coating material was found by said analysis. SEM analysis of the breakage surface after adhesion testing underline the influence of microstructure on the mechanical properties. Since enhanced amount of laterally expanded defects in the microstructure lead to rather cohesive break, SEM images exhibit an enhanced amount of remaining coating material on the aluminum substrate at position $40 \mathrm{~mm}$. Consequently, microstructure analysis at measurement position $100 \mathrm{~mm}$ show almost no laterally expanded splat interface as well as lowered amount of coating material on the substrate.

\section{Conclusion}

Three major methods are used to evaluate the thermal diffusivity as well as microstructural and mechanical properties of thermally sprayed cylinder bore coatings. Since thermal diffusivity, adhesion strength as well as amount, size and shape of coating defects change significantly and systematically along the cylinder liner, analysis of the used methods disclose direct influence of microstructural properties on both, thermal diffusivity and adhesion strength. Hence, TWI measurements open the ability to analyze the defect distribution in the microstructure of the cylinder coating. Further, the shown analysis reveal TWI measurements to non-destructively evaluate the adhesion strength of thermally sprayed cylinder bore coatings due to the underlying microstructure.

\section{REFERENCES}

[1] M.Schmidt, H. Spieth, J. Bauer, C. Haubach, Prozesskette NANOSLIDE, in: 100 Betriebe für Ressourceneffizienz-Band 1, Springer, 2017, pp. 234-237.

[2] B. Schilder, A. Garling, F. Reimer, M. Hamann, R. Joos, J. Hüger, M. Pöhlmann, T. Lampke, CFD enhanced thermal spray process for coating of cylinder bore of car engines, Journal of Thermal Spray Technology, Current status: submitted, review process, (2019).

[3] J. Arbogast, R. Schaller, S. Lindemann, B. Schilder, M. Rohde, H.J. Seifert, Thermal wave interferometry measurements and microstructural analysis of twin wire arc spray cylinder coatings for passenger car engines, Infrared Physics and Technology, Current status: submitted, review process, (2019).

[4] S. Deshpande, S. Sampath, H. Zhang, Mechanisms of oxidation and its role in microstructural evolution of metallic thermal spray coatings-case study for ni-al, Surface and Coatings Technology 200 (18-19) (2006) 53955406.

[5] P. Patel, D. Almond, Thermal wave testing of plasma-sprayed coatings and a comparison of the effects of coating microstructure on the propagation of thermal and ultrasonic waves, Journal of Materials Science 20 (3) (1985) 955-966.

[6] C. Bennett, R. Patty, Thermal wave interferometry: a potential application of the photoacoustic effect, Applied Optics 21 (1) (1982) 49-54.

[7] F. Cernuschi, S. Ahmaniemi, P. Vuoristo, T. Mäntylä, Modelling of thermal conductivity of porous materials: application of thick thermal barrier coatings, Journal of the European Ceramic Society 24 (9) (2004) 2657-2667.

[8] L. Pawlowski, P. Fauchais, Thermal transport properties of thermally sprayed coatings, International materials reviews 37 (1) (1992) 271-289. 